\title{
Glutamate dehydrogenase is a novel prognostic marker and predicts metastases in colorectal cancer patients
}

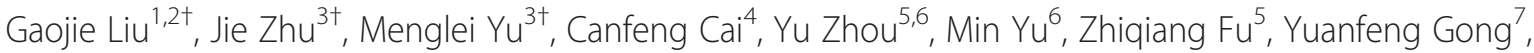
Bin Yang ${ }^{1}$, Yingru Li ${ }^{1}$, Quanbo Zhou ${ }^{5}$, Qin Lin ${ }^{5}$, Huilin Ye ${ }^{5}$, Liangtao Ye ${ }^{5}$, Xiaohui Zhao ${ }^{8}$, Zhihua Li ${ }^{8}$, Rufu Chen ${ }^{5}$, Fanghai Han ${ }^{1}$, Chaoming Tang ${ }^{4^{*}}$ and Bing Zeng ${ }^{2,4,5^{*}}$

\begin{abstract}
Background: Glutamate dehydrogenase $(\mathrm{GDH})$ is a key enzyme that catalyzes the final reaction of the glutamine metabolic pathway, and has been reported implicated in tumor growth and metastasis. However, it's clinical significance and role in colorectal cancer (CRC) pathogenesis is largely unknown.

Methods: The expression of GDH was determined by qPCR, western blot and immunohistochemistry in CRC cells and samples. The correlation of GDH expression with clinicopathologic features and prognosis was analyzed. The functional role of GDH in CRC cell proliferation, motility and metastasis was evaluated.

Results: We found that GDH was up-regulated both in colorectal cancer and metastatic lesions $(n=104)$. Patients with high GDH expression had poorer overall survival (HR 2.32; $95 \% \mathrm{Cl} 1.26-4.26 ; P=0.007)$ and poorer disease-free survival rates (HR 2.48; 95\% Cl 1.25-4.92; $P=0.009$ ) than those with low GDH expression. Furthermore, we showed that GDH expression was an independent prognostic factor for CRC. In addition, over-expression of GDH promoted cell proliferation, migration and invasion in vitro, whereas loss function of GDH did the opposite. Finally, we demonstrated that the promotion of CRC progression by GDH correlated with activation of STAT3 mediated epithelial-mesenchymal transition (EMT) induction.
\end{abstract}

Conclusions: These results indicate that GDH plays a critical role in CRC progression, and may provide a novel metabolism therapeutic target for CRC treatment.

Keywords: Colorectal cancer, Prognosis, GDH, STAT3, EMT

\section{Introduction}

Colorectal cancer (CRC) is one of the leading causes of cancer mortality in most courtiers, and globally affects over a million people each year in the developed countries [1]. Distant metastasis through lymphatic or hematogenous dissemination contributes to a poor prognosis for CRC, and the liver is the most frequent site of distant metastasis of CRC [2].

\footnotetext{
* Correspondence: chaomingtt001@163.com; zengbing2007@163.com ${ }^{\dagger}$ Equal contributors

${ }^{4}$ Department of Gastrointestinal Surgery, Qingyuan People's Hospital, The sixth affiliated hospital of Guangzhou Medical University, Guangdong 511518, China

${ }^{2}$ Key Laboratory of Malignant Tumor Gene Regulation and Target Therapy of Guangdong Higher Education Institutes, Sun Yat-sen Memorial Hospital of Sun Yat-sen University, Guangzhou 510120, China

Full list of author information is available at the end of the article
}

Currently, liver resection remains a standard procedure and the only potentially curative therapy for colorectal liver metastases (CLM). Unfortunately, the initial resection rates are reported to be less than $25 \%$ [3], with a high recurrence rate of $70-80 \%$ after curative resection [4]. Chemotherapy alone or in addition to local minimally invasive treatment, such as radiofrequency ablation, transarterial chemotherapy, or percutaneous ethanol injection, is the most treatment options in those who are not suitable for resection [5]. However, the underlying mechanism of this aggressive biology of CRC is largely unknown.

Aberrant energy metabolism is a critical hallmark for many types of human tumors [6]. Increasingly evidences have shown that glutamine metabolism plays key roles

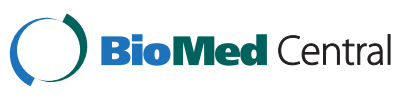


in tumor growth and invasion, and contributes poor outcomes [7-9]. Glutamine is first catabolyzed to glutamate and than to generate a-ketoglutarate, a tricarboxylic acid (TCA) cycle intermediate. Glutaminolysis supports the viability of cancer cells by supporting ATP production and by the biosynthesis of proteins, lipids, and nucleotides, and suppress oxidative stress through glutathione synthesis [10]. More importantly, oncogenes and tumor suppressors, such as SIRT4, mTORC1, K-RAS and p53, have been implicated in the regulation of glutamine metabolism [11-14]. Accordingly, it positively regulates the mTORC1 pathway by facilitating the uptake of leucine [15], and regulates STAT3 pathway by promoting tyrosine Y705 phosphorylation [9].

Glutamate dehydrogenase (GDH) is an enzyme that plays a pivotal role in glutamine metabolism by converting glutamate to a-ketoglutarate, especially when glucose is insufficient or under hypoxia. Recently, Csibi and coworkers [16] reported that mTORC1 promoted glutaminolysis by activating GDH to facilitate cell proliferation, transformation, and tumor development by repressing SIRT4. Lorin and colleagues [15] found that GDH contributed to autophagy by activating mTORC1 and by limiting the formation of reactive oxygen species in transformed cells. Yang [17] demonstrated that GDH activity is required for glioblastoma cell to survive impairments of glycolysis brought about by glucose deprivation. Moreover, studies found that treatment with epigallocatechin gallate (EGCG), an allosteric inhibitor of GDH, has considerable effect on tumor growth $[18,19]$. However, the clinical significance and role of GDH expression in colorectal cancer has not yet been investigated.

In the present study, we examined the expression of GDH in CRC and further analyzed the clinical significance of $\mathrm{GDH}$ in a cohort of CRC patients. In addition, we explored the potential role of GDH in CRC cell proliferation and motility, which could help to better understand the pathogenesis of CRC and may further provide a novel therapeutic target for the treatment of CRC patients.

\section{Materials \\ Cell culture, reagents and Lentiviral transduction}

The human colon cancer cell lines HCT116, DLD-1, SW480, RKO and LoVo were grown in RPMI-1640 medium supplemented with $10 \%$ fetal bovine serum (FBS). The human colon epithelial cell line NCM460 was cultured in MEM medium supplemented with $10 \%$ FBS. All cell lines were maintained at $37^{\circ} \mathrm{C}$ in a humidified atmosphere with $5 \% \mathrm{CO} 2$.

AG490 and DMSO were obtained from Sigma. The GDH short hairpin RNA (shRNA) was synthesized and cloned into a pLKO.1-TRC vector (Addgene). These vectors were co-transfected into $293 \mathrm{~T}$ cells along with the retroviral packaging plasmid. After transfection, the supernatants were harvested and used to infect CRC cells, and the stably transfected cells were selected with puromycin according the manufacturer's protocol.

\section{Tumor specimens}

Twenty freshly frozen CRC samples and corresponding non tumor tissues were obtained from Sun Yat-sen Memorial Hospital of Sun Yat-sen University. In addition, we collected 104 paraffin embedded CRC specimens from our hospital between January 2002 and February 2005. Tumor staging for the specimens was carried out according to the American Joint Committee on Cancer staging criteria. The median follow-up time was 62.5 months (range from 6.7 to 99). The patients' overall survival (OS) and disease-free survival (DFS) durations were defined as the interval from initial surgery to death and from initial surgery to clinically proven recurrence or metastasis respectively. The study was approved by the Institute Research Ethics Committee at the Sun Yat-sen University, and written informed consent was obtained from each patient.

\section{Immunohistochemical analysis}

Sections of paraffin-embedded CRC specimens were prepared and standard immunohistochemical procedures were carried out as previously described [20]. Briefly, samples were deparaffinized and rehydrated, and the endogenous peroxidase activity was quenched. Antigen retrieval was performed, and the sections were blocked with bovine serum albumin and then incubated with antiGDH antibody (Abcam; 1:200). Sections were washed and then incubated with a biotinylated secondary antibody and visualized with 3,3-diaminobenzidine.The staining results were scored by two pathologists blinded to the clinical data. Staining index was calculated as the product of the staining intensity ( 0 , no staining; 1 , weak staining; 2 , moderate staining; 3 , strong staining) and the proportion of positive cells ( 0 , no positive tumor cells; $1,<10 \%$; $2,10-35 \% ; 3,35-70 \% ; 4,>70 \%)$. The immunoreactivity score (IRS) was resulted from the multiplication of both parameters. Using this method of assessment, we evaluated GDH expression by determining the IRS, with scores of $0,1,2,3,4,6,8,9$ or 12 . The samples were divided into 2 groups as follows: low (IRS $=0-4)$, and high (IRS $\geq 6$ ).

\section{RNA extraction and quantitative real-time PCR}

Total RNA was extracted using TRIzol reagent. The reverse-transcription PCR (RT-PCR) was performed using transcriptase, and the quantitative real-time PCR (qRTPCR) was performed in a LightCycler480 System using a SYBR Premix ExTaq kit according to the manufacturer's instructions. Primers for qRT-PCR are as follows. GDH: Forward, 5'-GGG ATT CTA ACT ACC ACT TGC TCA3', Reverse 5'-AAC TCT GCC GTG GGT ACA AT-3'. 
GAPDH: Forward, 5'-CTC CTC CTG TTC GAC AGT CAG C-3', Reverse, 5'-CCC AAT ACG ACC AAA TCC GTT-3'. The relative expression levels were calculated by the $2^{-\Delta \Delta} \mathrm{CT}$ method. Each assay was carried out in triplicate.

\section{Western blot analysis}

Cell cytosolic protein fractions were prepared using RIPA buffer. According to standard Western blot procedures, briefly, proteins were separated by SDS-PAGE and then transferred to polyvinylidene fluoride (PVDF) membranes. After blocking in 5\% nonfat milk, the membranes were incubated with the following primary antibodies: GDH antibody (Abcam), STAT3, pSTAT3 (Tyr705), E-Cadherin, Vimentin, ZEB1 and GAPDH antibody (Cell Signaling Technology) according to the manufacturer's instructions.

\section{Cell proliferation}

Cell proliferation was analysed with the MTT assay. Cells were seeded in 96-well plates at a density of 1,000 cells per well. At 1, 2, 3 and 4 days, the cells were stained with $20 \mu \mathrm{l}$ of MTT $(0.5 \mathrm{mg} / \mathrm{ml})$ for $4 \mathrm{~h}$, and after which the medium was removed, and $100 \mu \mathrm{l}$ of DMSO was added. The absorbance was measured at $490 \mathrm{~nm}$. The anchorage-independent sphere formation assay was performed by culturing the cells in suspension in serumfree DMEM-F12 supplemented with B27 (Invitrogen), EGF (BD Biosciences, CA, USA), 0.4\% bovine serum albumin (Sigma, MO, USA), and insulin (Sigma).

\section{Cell migration and invasion assays}

Cell motility was assessed by wound healing assay as previously described [21]. Results were expressed as a migration index: the distance migrated by targeted relative to the distance migrated by control cells. Cell invasion assays were performed using 24-well transwells ( $8-\mu \mathrm{m}$ pore size, BD Sciences) coated with matrigel $(1 \mathrm{mg} / \mathrm{ml}$, BD Sciences), as previously described [21]. The inserts were stained with $0.2 \%$ crystal violet, imaged, and counted under an inverted microscope in six randomly selected fields. All experiments were carried out in triplicate and repeated at least three times.

\section{Statistical analysis}

Data are presented as the mean $\pm \mathrm{SD}$, and differences between groups were analyzed using Student's t-test or chi-squared test or fisher exact test. The Kaplan-Meier method and log-rank test were used to estimate survival rates. Cox proportional hazards model was used to calculate univariate and multivariate hazard ratios for the study variables. Statistical analyses were performed with SPSS 16.0 software (Chicago, IL), and P values of $<0.05$ were considered statistically significant.

\section{Results}

\section{Expression of GDH in CRC cells and tissues}

Quantitative RT-PCR and western blot were used to determine the levels of GDH mRNA and protein in CRC cell lines. As shown in Figure 1A and B, GDH was overexpressed in CRC cell lines when compared to human colon epithelial cell line NCM460. LoVo $(P=0.0003)$, HCT116 $(P=0.0036)$, HCT116 $(P=0.0019)$. Next, we analyzed GDH mRNA expression in 20 freshly frozen CRC tissues and corresponding non tumor tissues. We found that GDH expression levels were considerably over-expressed in CRC when compared to corresponding non tumor tissues (Figure 1C). Moreover, GDH protein expression levels were also up-regulated in tumor tissues (Figure 1D).

\section{GDH over-expression is associated with CRC metastasis}

To determine the effect of GDH expression on CRC progression, we next analyzed the expression of GDH protein in a set of 104 paraffin embedded CRC tissue using immunohistochemistry. Representative staining of GDH in CRC tissue is shown in Figure 2. We observed that GDH was highly expressed in 56 of the 104 (53.8 \%) patients, and GDH-positive staining was mainly in the cytoplasm of the cancer cells. Interestingly, immunostaining results showed that metastatic lymph nodes and liver metastases lesions had higher levels of GDH expression (Figure 2E and F).

Furthermore, patients with high GDH expression exhibited a significant association with tumor size $(P=0.046)$, tumor stage $(P=0.001)$, lymph node metastasis $(P=0.001)$ and liver metastasis $(P=0.021)$. While there were no significant associations between GDH expression and age, sex, location, grade of differentiation, T stage, CEA, venous invasion, or nervous invasion (Table 1).

\section{GDH over-expression is association with poor prognosis in CRC patients}

To assess the clinical significance of GDH over-expression in CRC, Kaplan-Meier analysis and the log-rank test were used to analyze the relationship between GDH expression and patient survival. We found that the 5 -year overall survival (OS) was significantly lower in patients with high GDH expression than in those with low GDH expression (38.1\% vs. $65.1 \%$; HR 2.32; 95\% CI 1.26-4.26; $P=0.007$ ) (Figure 3A). Moreover, patients with high GDH expression were significantly associated with poorer disease-free survival (DFS) rates than those with low GDH expression (HR 2.48; 95\% CI 1.25-4.92; $P=0.009$ ) (Figure 3B). In addition, multivariate analyses indicated that GDH expression, lymph node metastasis, and liver metastasis were independent prognostic indicators for both OS and DFS in CRC patients (Table 2). 


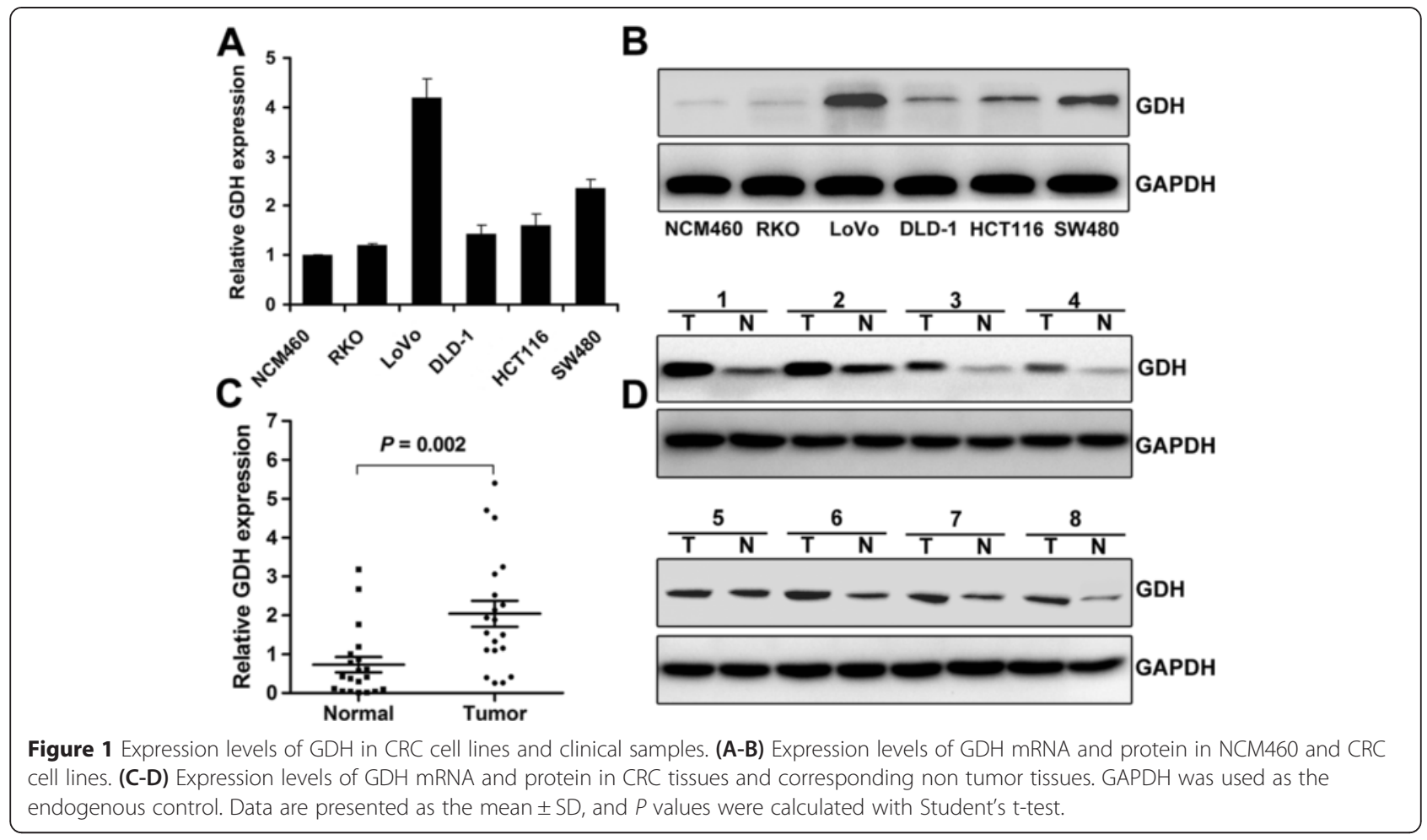

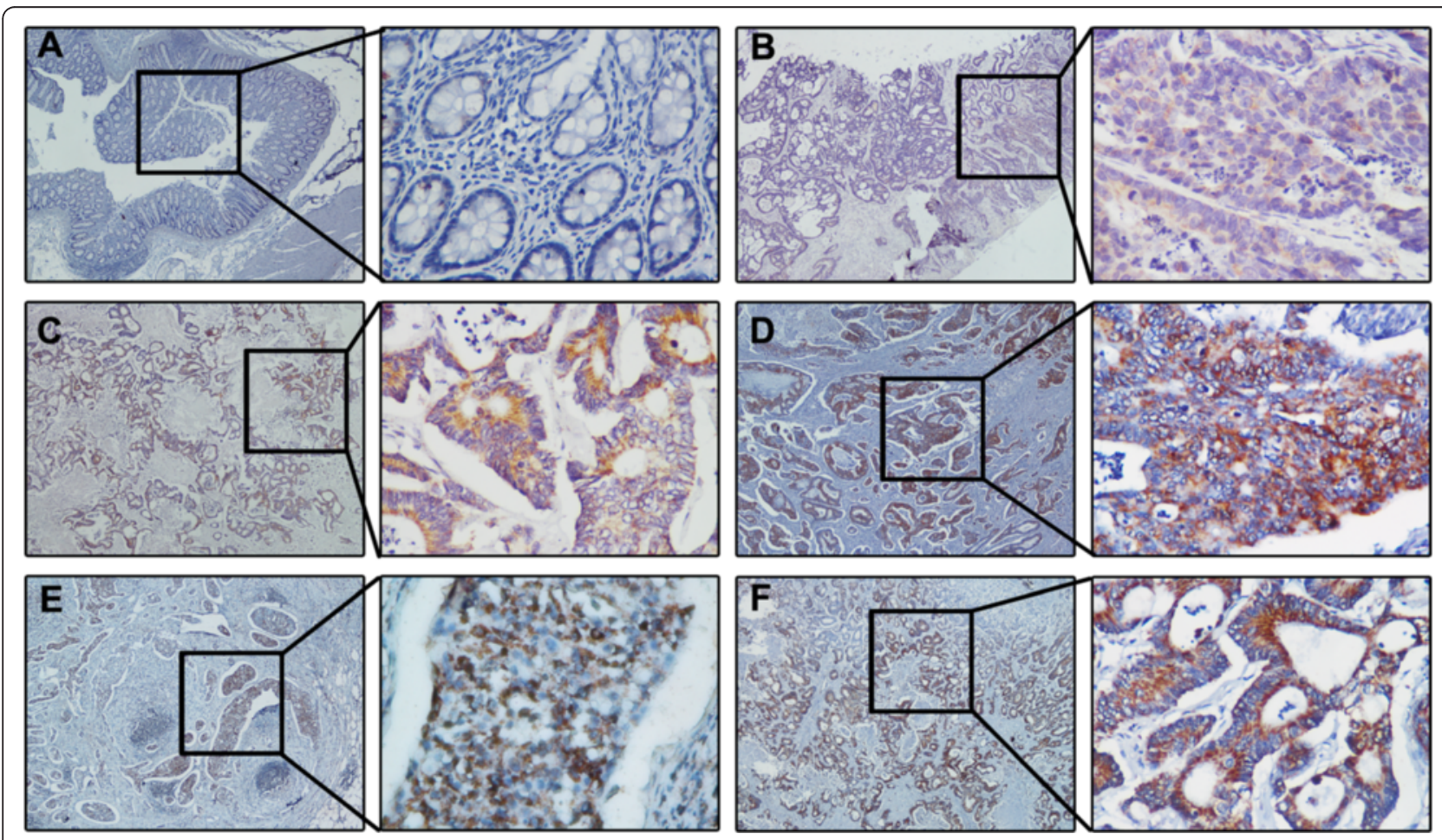

Figure 2 Expression levels of GDH in CRC tissues, metastatic lymph nodes and liver metastases lesions. Representative images of CRC tissues with GDH Negative staining (A), Weak staining: light yellow (B), moderate staining: yellow brown (C), strong staining: brown (D). Representative images of GDH immunostaining in metastatic lymph nodes (E) and liver metastases lesions (F). (Envision $\times 40, \times 400$ ). 
Table 1 Clinicopathological factors and GDH expression in 104 colorectal cancers

\begin{tabular}{|c|c|c|c|c|c|}
\hline \multirow[t]{2}{*}{ Factor } & \multicolumn{2}{|c|}{ GDH high expression } & \multicolumn{2}{|c|}{ GDH low expression } & \multirow[t]{2}{*}{$P$-value } \\
\hline & \multicolumn{2}{|c|}{$n=56, n(\%)$} & \multicolumn{2}{|c|}{$n=48, n(\%)$} & \\
\hline \multicolumn{6}{|l|}{ Age } \\
\hline$<60$ & 27 & 48.2 & 26 & 54.2 & 0.545 \\
\hline$\geq 60$ & 29 & 51.8 & 22 & 45.8 & \\
\hline \multicolumn{6}{|l|}{ Gender } \\
\hline Male & 34 & 60.7 & 27 & 56.2 & 0.645 \\
\hline Female & 22 & 39.3 & 21 & 43.8 & \\
\hline \multicolumn{6}{|l|}{ Location } \\
\hline Colon & 33 & 58.9 & 32 & 66.7 & 0.416 \\
\hline Rectum & 23 & 41.1 & 16 & 33.3 & \\
\hline \multicolumn{6}{|l|}{ Tumor size } \\
\hline$<3 \mathrm{~cm}$ & 24 & 42.9 & 30 & 62.5 & $0.046^{*}$ \\
\hline$\geq 3 \mathrm{~cm}$ & 32 & 57.1 & 18 & 37.5 & \\
\hline \multicolumn{6}{|l|}{ CEA } \\
\hline Normal & 26 & 46.4 & 25 & 52.1 & 0.656 \\
\hline Elevated & 30 & 53.6 & 23 & 47.9 & \\
\hline \multicolumn{6}{|c|}{ Grade of differentiation } \\
\hline Well & 11 & 19.6 & 14 & 29.2 & 0.296 \\
\hline Moderate & 35 & 62.5 & 27 & 56.3 & \\
\hline Poor & 10 & 17.9 & 7 & 14.5 & \\
\hline \multicolumn{6}{|c|}{ Depth of invasion } \\
\hline $\mathrm{T} 1$ and $\mathrm{T} 2$ & 31 & 55.4 & 26 & 54.2 & 0.903 \\
\hline $\mathrm{T} 3$ and $\mathrm{T} 4$ & 25 & 44.6 & 22 & 45.8 & \\
\hline \multicolumn{6}{|c|}{ Tumor stage } \\
\hline | and || & 14 & 25 & 28 & 58.3 & $0.001 *$ \\
\hline III and IV & 42 & 75 & 20 & 41.7 & \\
\hline \multicolumn{6}{|c|}{ Lymph node metastasis } \\
\hline Negative & 20 & 35.7 & 33 & 68.8 & $0.001 *$ \\
\hline Positive & 36 & 64.3 & 15 & 31.2 & \\
\hline \multicolumn{6}{|c|}{ Liver metastasis } \\
\hline Negative & 40 & 71.4 & 43 & 89.6 & $0.021 *$ \\
\hline Positive & 16 & 28.6 & 5 & 10.4 & \\
\hline \multicolumn{6}{|c|}{ Venous invasion } \\
\hline Negative & 50 & 89.3 & 45 & 93.8 & 0.501 \\
\hline Positive & 6 & 10.7 & 3 & 6.2 & \\
\hline \multicolumn{6}{|c|}{ Nervous invasion } \\
\hline Negative & 36 & 64.3 & 38 & 79.2 & 0.095 \\
\hline Positive & 20 & 35.7 & 10 & 20.8 & \\
\hline
\end{tabular}

GDH knockdown impairs CRC cell proliferation and motility in vitro

To confirm the functional role of GDH in CRC cells, we first transfected shGDH or scrambled plasmid into CRC cells (Additional file 1: Figure S1), and found that $\mathrm{GDH}$ depletion significantly suppressed cell proliferation and inhibited anchorage-independent growth compared to the controls (Figure 4A and B). Next, the wound healing assay showed that downregulation of GDH led to a marked decrease in cell motility than that of control cells (Figure 4C). Similarly, the transwell invasion assay demonstrated that 

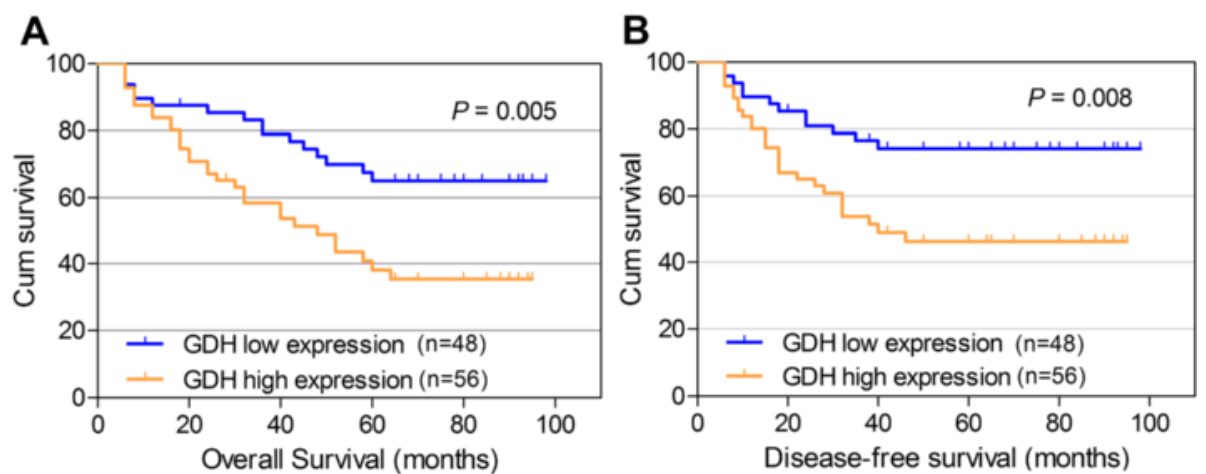

Figure 3 Kaplan-Meier curves for OS and DFS. Patients with high GDH expression had poorer overall survival (A) and poorer disease-free survival (B) rates than patients with low GDH expression.

GDH depletion resulted in a significant reduction of cell invasion (Figure 4D).

\section{GDH promotes CRC cell motility via STAT3 mediated EMT Induction}

Epithelial-mesenchymal transition (EMT) contributes to tumor invasion and metastasis in many cancers including CRC [22]. Previous studies found that glutamine metabolism could promote tumor growth and invasion through STAT3 pathway, a regulator of EMT and aberrantly activated in CRC [9]. These studies prompted us to ask whether GDH promotes CRC cell motility through STAT3 mediated EMT Induction. Interesting, we found that knockdown of GDH significant attenuated STAT3 phosphorylation, and decreased Vimentin and ZEB1 expression, while up-regulated E-cadherin expression in CRC cells (Figure 5A). Furthermore, blocked STAT3 pathway by AG490 significantly increased E-cadherin and decreased ZEB1 and Vimentin expressions in CRC cells (Figure 5A). Additionally, STAT3 pathway blockage could significant inhibited cell proliferation (Figure 5B), and attenuated the migration and invasion ability of CRC cells (Figure 5C and D).

To further prove this hypothesis, we establish GDHoverexpression SW480 cells, and found that GDHoverexpression could promote STAT3 phosphorylation, and up-regulate Vimentin and ZEB1 expression, while decrease E-cadherin expression (Additional file 2: Figure S2). Furthermore, AG490 treatment could significantly increase E-cadherin, and decrease ZEB1 and Vimentin expressions in GDH-overexpression SW480 cells (Additional file 2: Figure S2). Taken together, these results suggested that GDH promotes CRC cell motility via STAT3 mediated EMT induction.

Table 2 Univariate and multivariable Cox regression analyses for overall and disease-free survival

\begin{tabular}{|c|c|c|c|c|c|c|c|c|}
\hline \multirow[t]{2}{*}{ Factor } & \multicolumn{4}{|c|}{ Univariate analysis } & \multicolumn{4}{|c|}{ Multivariate analysis } \\
\hline & HR & \multicolumn{2}{|c|}{$95 \% \mathrm{Cl}$} & $P$-value & HR & \multicolumn{2}{|c|}{$95 \% \mathrm{Cl}$} & $P$-value \\
\hline \multicolumn{9}{|l|}{ Overall survival } \\
\hline Depth of invasion (T1-2/T3-4) & 2.07 & 1.16 & 3.69 & $0.014^{*}$ & 1.82 & 0.98 & 3.38 & 0.060 \\
\hline Tumor stage (I-|I/III-IV) & 2.01 & 1.11 & 3.63 & $0.021^{*}$ & 1.37 & 0.72 & 2.6 & 0.333 \\
\hline Grade of differentiation (well, moderate/poor) & 2.13 & 1.01 & 4.45 & $0.046^{*}$ & 1.28 & 0.53 & 3.05 & 0.581 \\
\hline Lymph node metastasis (negative/positive) & 4.68 & 2.45 & 8.94 & $<0.001^{*}$ & 3.47 & 1.66 & 7.27 & $0.001^{*}$ \\
\hline Liver metastasis (negative/positive) & 3.53 & 1.88 & 6.63 & $<0.001^{*}$ & 2.62 & 1.29 & 5.32 & $0.008^{*}$ \\
\hline Venous invasion (negative/positive) & 2.45 & 1.04 & 5.8 & $0.042^{*}$ & 1.66 & 0.64 & 4.32 & 0.294 \\
\hline Nervous invasion (negative/positive) & 2.25 & 1.25 & 4.05 & $0.007^{*}$ & 1.14 & 0.59 & 2.19 & 0.703 \\
\hline GDH (low/high) & 2.32 & 1.26 & 4.26 & $0.007^{*}$ & 2.42 & 1.23 & 4.78 & $0.011^{*}$ \\
\hline \multicolumn{9}{|l|}{ Disease-free survival } \\
\hline Lymph node metastasis (negative/positive) & 5.73 & 2.68 & 12.28 & $<0.001^{*}$ & 4.70 & 2.08 & 10.59 & $<0.001^{*}$ \\
\hline Liver metastasis (negative/positive) & 3.81 & 1.95 & 7.46 & $<0.001^{*}$ & 2.38 & 1.16 & 4.87 & $0.018^{*}$ \\
\hline GDH (low/high) & 2.48 & 1.25 & 4.92 & $0.009^{*}$ & 2.17 & 1.05 & 4.47 & $0.037^{*}$ \\
\hline
\end{tabular}

HR relative risk, $95 \% \mathrm{Cl}$ 95\% confidence interval.

*Statistically significant $P<0.05$, Cox proportional hazard regression model. 


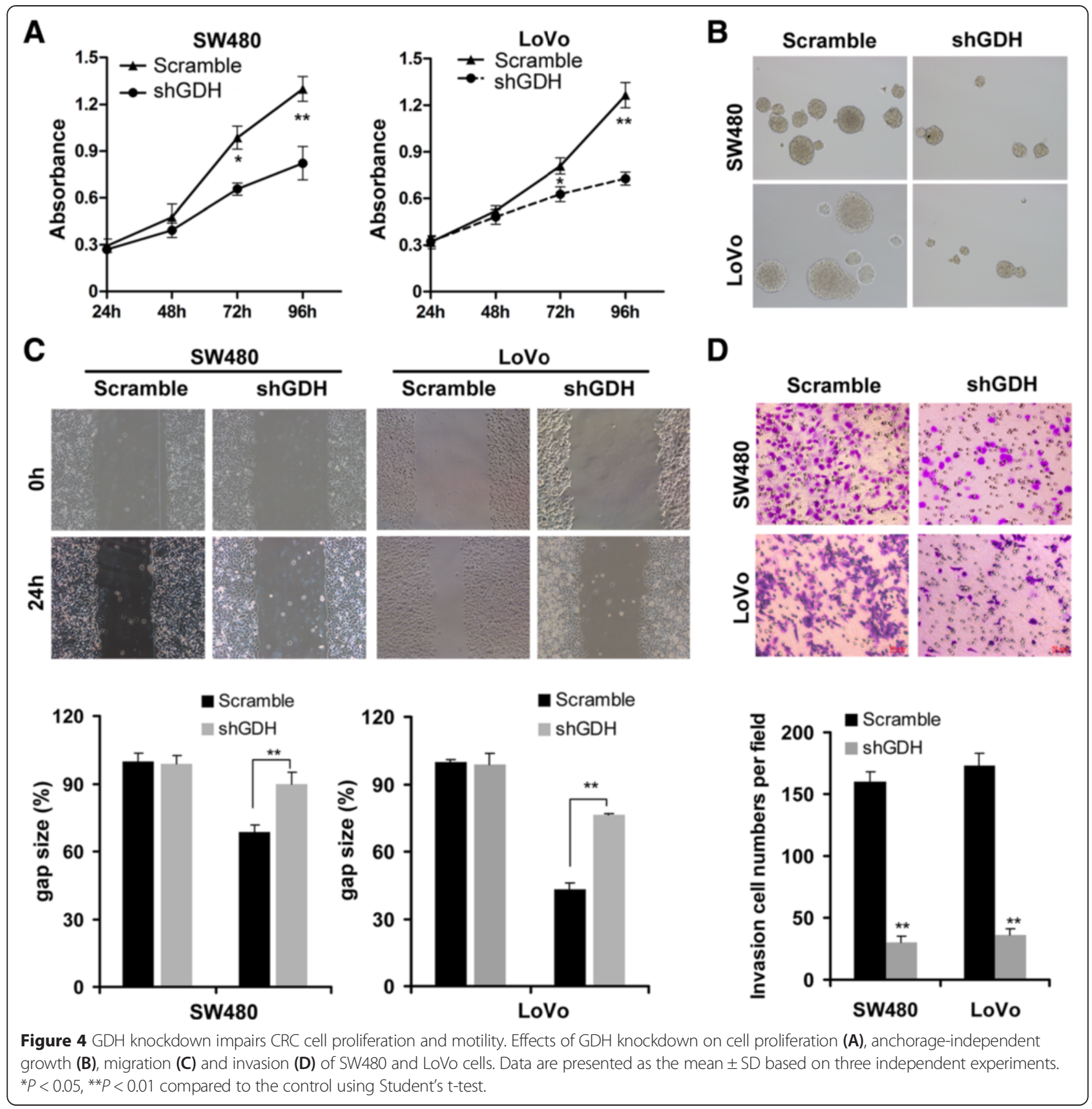

\section{Discussion}

Distant metastasis is a major cause of death in CRC patients [23]. In this study, we found that GDH was up-regulated in both CRC cells and clinical samples, and correlated with poor survival and liver metastasis of CRC. Importantly, our results revealed that $\mathrm{GDH}$ is an independent prognostic factor for survival, which might served as valuable prognosis markers for CRC. Finally, we demonstrated that GDH promotes CRC cell motility via STAT3 mediated EMT Induction. We show here for the first time that GDH plays important roles in the metastatic and aggressive biology of CRC, which might served as a predictive marker for CRC prognosis, and presents a viable metabolic regulation strategy for CRC.

Metabolism is now recognized as a key feature of the cancer cells, and increasingly evidence has linked cell metabolism with cancer outcome [24-26]. Although intensive studies have focused on the role of glutamine metabolism in regulating growth and survival [27-29], its role in motility and metastasis of cancers is not well understood. Recently, Yang [9] revealed that high invasive OVCA cells were strongly glutamine dependent, and glutamine was a key mitochondrial substrate for driving cancer metastasis by induction of STAT3 serine 


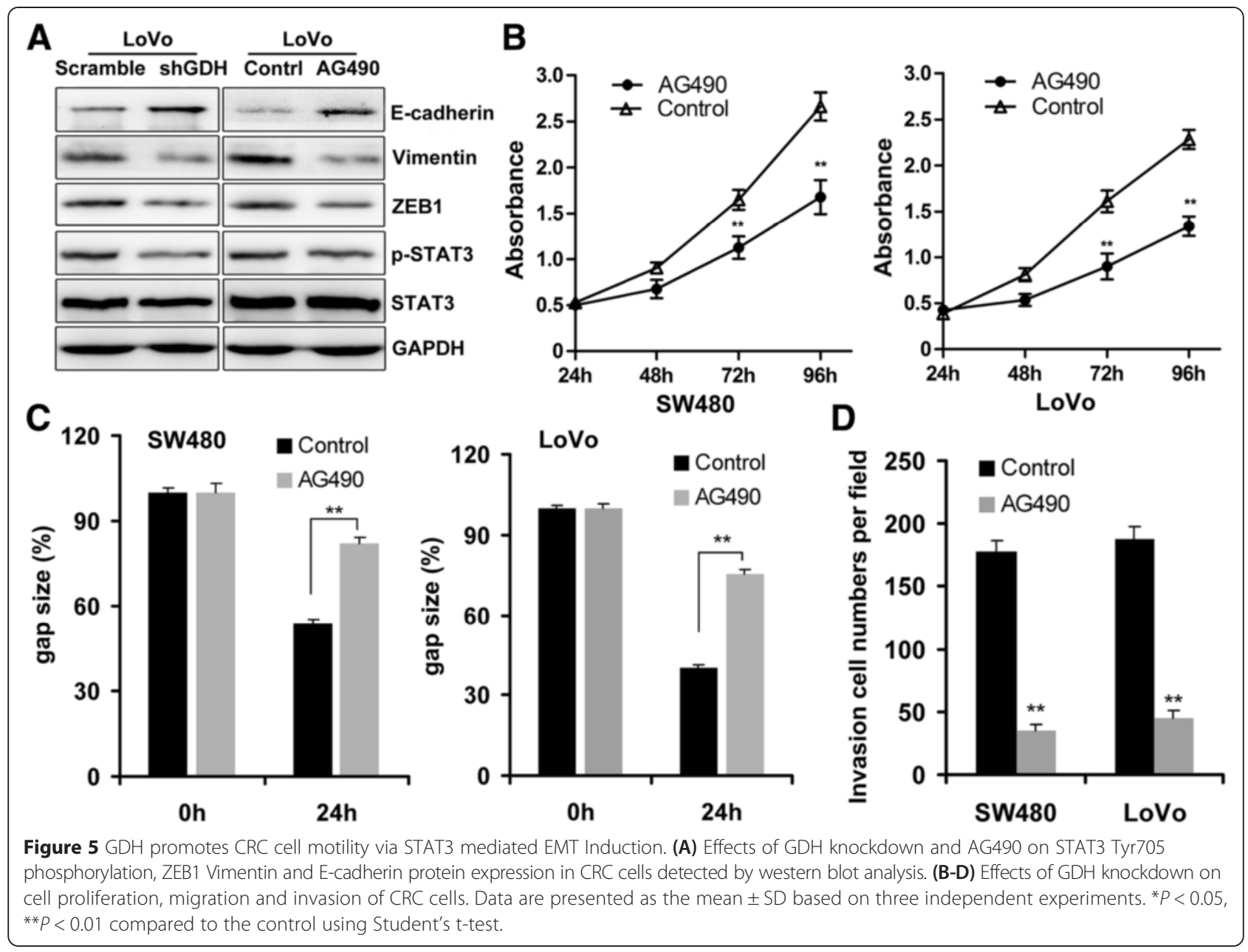

phosphorylation. Moreover, glutamine restriction inhibited melanoma cell migration via modulation of actin cytoskeleton remodeling [30], and GDH activity was required for glioblastoma cell to survive impairments of glycolysis brought about by glucose deprivation [17]. In the present study, we focus on the role of GDH, a key enzyme converting glutamate to a-ketoglutarate then entry into the TCA cycle. We found a high expression of GDH in aggressive CRC cell lines, and altered GDH expression affected cell proliferation, migration and invasion. More importantly, we found direct clinical evidence of a strong correlation between GDH overexpression and unfavorable clinicopathologic variables such as tumor size, tumor stage, lymph node metastasis and liver metastasis. These findings robustly suggested that GDH plays an important role in CRC progression and metastasis.

It has been reported that approximately $30 \%$ of CRC patients presenting with synchronous metastases and $70 \%$ ultimately developing liver metastases, and $50 \%$ of CRC deaths are caused by liver metastases [31-33]. However, the mechanism of this aggressive biology of
CRC is largely unknown. Epithelial-mesenchymal transition (EMT) involves the loss of E-Cadherin mediated cell adhesion and polarity, and is proposed to be a crucial mechanism regulating the initial steps in metastatic progression [34]. Several studies have examined the possible role of EMT in CRC progression including liver metastases [35-37]. To our knowledge, few studies have described EMT with glutamine metabolism in CRC. In this study, we found that loss function of GDH lead to aberrant expression of EMT markers, and suppressed cell proliferation and motility of CRC cells. Together with previous studies, our findings further support the crucial role of EMT in CRC metastasis.

Evidences showed that metabolism enzyme themselves could be oncogenic by altering cell signaling that mediated multiple aspects of cancer metastatic biology $[15,28,38]$. Considering the crucial role of GDH involved in CRC metastases, it is necessary to elucidate the underlying mechanism of GDH mediated induction of EMT. STAT3, a key regulator of EMT, has been reported frequently activated in CRC [39-41]. Zhou [42] showed that STAT3 could induce PTTG expression to facilitate 
tumor growth and metastasis of CRC. Xiong [43] found that STAT3 down-regulated E-Cadherin expression via ZEB1 in colorectal cancer cells. In this study, we demonstrated that altered GDH expression significantly affected the tyrosine phosphorylation of STAT3, and GDH mediated invasiveness of CRC was STAT3 depended. Our results consist with previous findings that tyrosine phosphorylation levels of STAT3 are dependent on glutamine's entry into the TCA cycle.

\section{Conclusions}

In conclusion, this study provided evidence supporting the critical role of GDH in CRC progression and metastasis. Our findings uncover a novel molecular mechanism for CRC progression and metastasis and provide novel metabolism therapeutic targets and strategies to control CRC progression and metastasis.

\section{Additional files}

Additional file 1: Effects of GDH knockdown in CRC cells.

Additional file 2: GDH promotes EMT via STAT3 pathway.

\section{Abbreviations}

CRC: Colorectal cancer; GDH: Glutamate dehydrogenase; EMT: Epithelialmesenchymal transition; CLM: Colorectal liver metastases; OS: Overall survival; DFS: Disease-free survival; TCA cycle: Tricarboxylic acid cycle.

\section{Competing interests}

The authors declare that they have no competing interests.

\section{Authors' contributions}

BZ and CMT conceived and designed the study. GJL, JZ and MLY performed the experiments and wrote the manuscript. CFC, YZ, MY, ZQF and YFG analysed and interpreted data. BY, YRL, QBZ, QL, HLY, LTY, XHZ, ZHL, RFC and $\mathrm{FHH}$ contributed reagents, materials and helped to draft the manuscript. All authors read and approved the final manuscript.

\section{Acknowledgements}

This work was financially supported by the National Natural Science Foundation of China (No. 81401996), the Doctoral Scientific Fund Project of the Ministry of Education of China (No. 20130171110074), and the Medical Research Foundation of Guangdong province (No. A2014228).

\footnotetext{
Author details

'Department of Gastrointestinal Surgery, Sun Yat-sen Memorial Hospital of Sun Yat-sen University, Guangzhou 510120, China. ${ }^{2}$ Key Laboratory of Malignant Tumor Gene Regulation and Target Therapy of Guangdong Higher Education Institutes, Sun Yat-sen Memorial Hospital of Sun Yat-sen University, Guangzhou 510120, China. ${ }^{3}$ Department of Emergency Surgery, Sun Yat-sen Memorial Hospital of Sun Yat-sen University, Guangzhou 510120, China. ${ }^{4}$ Department of Gastrointestinal Surgery, Qingyuan People's Hospital, The sixth affiliated hospital of Guangzhou Medical University, Guangdong 511518, China. ${ }^{5}$ Department of Pancreaticobiliary Surgery, Sun Yat-sen Memorial Hospital of Sun Yat-sen University, Guangzhou 510120, China. ${ }^{6}$ Department of Pancreaticobiliary Surgery, Guangdong Academy of Medical Sciences and Guangdong General Hospital, Guangzhou 510120, China. ${ }^{7}$ Department of Hepatobiliary Surgery, Cancer Center of Guangzhou Medical University, Guangzhou 510120, China. ${ }^{8}$ Department of Oncology, Sun Yat-sen Memorial Hospital, Sun Yat-sen University, Guangzhou 510120, China.
}

Received: 1 December 2014 Accepted: 21 April 2015

Published online: 07 May 2015

\section{References}

1. DeSantis CE, Lin CC, Mariotto AB, Siegel RL, Stein KD, Kramer JL, et al. Cancer treatment and survivorship statistics, 2014. CA Cancer J Clin. 2014;64:252-71.

2. Poston GJ, Figueras J, Giuliante F, Nuzzo G, Sobrero AF, Gigot JF, et al. Urgent need for a new staging system in advanced colorectal cancer. J Clin Oncol. 2008;26:4828-33.

3. Bismuth $H$, Adam R, Levi F, Farabos C, Waechter F, Castaing D, et al. Resection of nonresectable liver metastases from colorectal cancer after neoadjuvant chemotherapy. Ann Surg. 1996;224:509-20. discussion 520-502.

4. Rees M, Tekkis PP, Welsh FK, O'Rourke T, John TG. Evaluation of long-term survival after hepatic resection for metastatic colorectal cancer: a multifactorial model of 929 patients. Ann Surg. 2008;247:125-35.

5. Abdalla EK, Vauthey JN, Ellis LM, Ellis V, Pollock R, Broglio KR, et al. Recurrence and outcomes following hepatic resection, radiofrequency ablation, and combined resection/ablation for colorectal liver metastases. Ann Surg. 2004;239:818-25. discussion 825-817.

6. Hanahan D, Weinberg RA. Hallmarks of cancer: the next generation. Cell. 2011;144:646-74.

7. McGuirk S, Gravel SP, Deblois G, Papadopoli DJ, Faubert B, Wegner A, et al. PGC-1alpha supports glutamine metabolism in breast cancer. Cancer Metab. 2013;1:22.

8. Timmerman LA, Holton T, Yuneva M, Louie RJ, Padro M, Daemen A, et al. Glutamine sensitivity analysis identifies the xCT antiporter as a common triple-negative breast tumor therapeutic target. Cancer Cell. 2013;24:450-65.

9. Yang L, Moss T, Mangala LS, Marini J, Zhao H, Wahlig S, et al. Metabolic shifts toward glutamine regulate tumor growth, invasion and bioenergetics in ovarian cancer. Mol Syst Biol. 2014;10:728.

10. Liu W, Le A, Hancock C, Lane AN, Dang CV, Fan TW, et al. Reprogramming of proline and glutamine metabolism contributes to the proliferative and metabolic responses regulated by oncogenic transcription factor c-MYC. Proc Natl Acad Sci U S A. 2012;109:8983-8.

11. Csibi A, Lee G, Yoon SO, Tong H, Ilter D, Elia I, et al. The mTORC1/S6K1 pathway regulates glutamine metabolism through the elF4B-dependent control of c-Myc translation. Curr Biol. 2014;24:2274-80.

12. Jeong SM, Xiao C, Finley LW, Lahusen T, Souza AL, Pierce $K$, et al. SIRT4 has tumor-suppressive activity and regulates the cellular metabolic response to DNA damage by inhibiting mitochondrial glutamine metabolism. Cancer Cell. 2013:23:450-63.

13. Son J, Lyssiotis CA, Ying H, Wang X, Hua S, Ligorio M, et al. Glutamine supports pancreatic cancer growth through a KRAS-regulated metabolic pathway. Nature. 2013;496:101-5.

14. Hu W, Zhang C, Wu R, Sun Y, Levine A, Feng Z. Glutaminase 2, a novel p53 target gene regulating energy metabolism and antioxidant function. Proc Natl Acad Sci U S A. 2010;107:7455-60.

15. Lorin S, Tol MJ, Bauvy C, Strijland A, Pous C, Verhoeven AJ, et al. Glutamate dehydrogenase contributes to leucine sensing in the regulation of autophagy. Autophagy. 2013;9:850-60.

16. Csibi A, Fendt SM, Li C, Poulogiannis G, Choo AY, Chapski DJ, et al. The mTORC1 pathway stimulates glutamine metabolism and cell proliferation by repressing SIRT4. Cell. 2013;153:840-54.

17. Yang C, Sudderth J, Dang T, Bachoo RM, McDonald JG, DeBerardinis RJ. Glioblastoma cells require glutamate dehydrogenase to survive impairments of glucose metabolism or Akt signaling. Cancer Res. 2009;69:7986-93.

18. Khan N, Bharali DJ, Adhami VM, Siddiqui IA, Cui H, Shabana SM, et al. Oral administration of naturally occurring chitosan-based nanoformulated green tea polyphenol EGCG effectively inhibits prostate cancer cell growth in a xenograft model. Carcinogenesis. 2014;35:415-23.

19. Shukla R, Chanda N, Zambre A, Upendran A, Katti K, Kulkarni RR, et al. Laminin receptor specific therapeutic gold nanoparticles (198AuNP-EGCg) show efficacy in treating prostate cancer. Proc Natl Acad Sci U S A. 2012;109:12426-31.

20. Guo N, Chen R, Li Z, Liu Y, Cheng D, Zhou Q, et al. Hepatitis C virus core upregulates the methylation status of the RASSF1A promoter through regulation of SMYD3 in hilar cholangiocarcinoma cells. Acta Biochim Biophys Sin (Shanghai). 2011;43:354-61.

21. Zeng B, Li Z, Chen R, Guo N, Zhou J, Zhou Q, et al. Epigenetic regulation of miR-124 by hepatitis $C$ virus core protein promotes migration and invasion of intrahepatic cholangiocarcinoma cells by targeting SMYD3. FEBS Lett. 2012;586:3271-8.

22. Takahashi Y, Sawada G, Kurashige J, Uchi R, Matsumura T, Ueo H, et al. Paired related homoeobox 1, a new EMT inducer, is involved in metastasis and poor prognosis in colorectal cancer. Br J Cancer. 2013;109:307-11. 
23. Loupakis F, Cremolini C, Masi G, Lonardi S, Zagonel V, Salvatore L, et al. Initial therapy with FOLFOXIRI and bevacizumab for metastatic colorectal cancer. N Engl J Med. 2014;371:1609-18.

24. Seyfried TN, Flores RE, Poff AM, D'Agostino DP. Cancer as a metabolic disease: implications for novel therapeutics. Carcinogenesis. 2014;35:515-27.

25. Chiu M, Tardito S, Pillozzi S, Arcangeli A, Armento A, Uggeri J, et al. Glutamine depletion by crisantaspase hinders the growth of human hepatocellular carcinoma xenografts. Br J Cancer. 2014;111:1159-67.

26. Grimm M, Cetindis M, Lehmann M, Biegner T, Munz A, Teriete $P$, et al. Association of cancer metabolism-related proteins with oral carcinogenesis - indications for chemoprevention and metabolic sensitizing of oral squamous cell carcinoma? J Transl Med. 2014;12:208.

27. Caneba CA, Bellance N, Yang L, Pabst L, Nagrath D. Pyruvate uptake is increased in highly invasive ovarian cancer cells under anoikis conditions for anaplerosis, mitochondrial function, and migration. Am J Physiol Endocrinol Metab. 2012;303:E1036-52.

28. Nomura DK, Long JZ, Niessen S, Hoover HS, Ng SW, Cravatt BF. Monoacylglycerol lipase regulates a fatty acid network that promotes cancer pathogenesis. Cell. 2010;140:49-61.

29. Cao Y, Rathmell JC, Macintyre AN. Metabolic reprogramming towards aerobic glycolysis correlates with greater proliferative ability and resistance to metabolic inhibition in CD8 versus CD4 T cells. PLoS One. 2014;9, e104104.

30. Fu YM, Zhang H, Ding M, Li YQ, Fu X, Yu ZX, et al. Specific amino acid restriction inhibits attachment and spreading of human melanoma via modulation of the integrin/focal adhesion kinase pathway and actin cytoskeleton remodeling. Clin Exp Metastasis. 2004;21:587-98.

31. Manfredi S, Lepage C, Hatem C, Coatmeur O, Faivre J, Bouvier AM. Epidemiology and management of liver metastases from colorectal cancer. Ann Surg. 2006;244:254-9.

32. Helling TS, Martin M. Cause of death from liver metastases in colorectal cancer. Ann Surg Oncol. 2014;21:501-6.

33. Landreau P, Drouillard A, Launoy G, Ortega-Deballon P, Jooste V, Come L, et al. Incidence and survival in late liver metastases of colorectal cancer. J Gastroenterol Hepatol. 2015;30:82-5.

34. Mirantes C, Espinosa I, Ferrer I, Dolcet X, Prat J, Matias-Guiu X. Epithelial-tomesenchymal transition and stem cells in endometrial cancer. Hum Pathol. 2013:44:1973-81.

35. Zhou Y, Wu J, Fu X, Du W, Zhou L, Meng X, et al. OTUB1 promotes metastasis and serves as a marker of poor prognosis in colorectal cancer. Mol Cancer. 2014;13:258.

36. Chanrion M, Kuperstein I, Barriere C, El Marjou F, Cohen D, Vignjevic D, et al. Concomitant Notch activation and p53 deletion trigger epithelial-tomesenchymal transition and metastasis in mouse gut. Nat Commun. 2014;5:5005.

37. Bai Z, Tai Y, Li W, Zhen C, Gu W, Jian Z, et al. Gankyrin activates IL-8 to promote hepatic metastasis of colorectal cancer. Cancer Res. 2013;73:4548-58.

38. Hamabe A, Konno M, Tanuma N, Shima H, Tsunekuni K, Kawamoto K, et al. Role of pyruvate kinase M2 in transcriptional regulation leading to epithelial-mesenchymal transition. Proc Natl Acad Sci U S A. 2014;111:15526-31.

39. Gulhati P, Bowen KA, Liu J, Stevens PD, Rychahou PG, Chen M, et al. mTORC1 and mTORC2 regulate EMT, motility, and metastasis of colorectal cancer via RhoA and Rac1 signaling pathways. Cancer Res. 2011;71:3246-56.

40. Rokavec M, Oner MG, Li H, Jackstadt R, Jiang L, Lodygin D, et al. IL-6R/ STAT3/miR-34a feedback loop promotes EMT-mediated colorectal cancer invasion and metastasis. J Clin Invest. 2014;124:1853-67.

41. Yang P, Li Z, Fu R, Wu H. Pyruvate kinase M2 facilitates colon cancer cell migration via the modulation of STAT3 signalling. Cell Signal. 2014;26:1853-62.

42. Zhou C, Tong Y, Wawrowsky K, Melmed S. PTTG acts as a STAT3 target gene for colorectal cancer cell growth and motility. Oncogene. 2014;33:851-61

43. Xiong H, Hong J, Du W, Lin YW, Ren LL, Wang YC, et al. Roles of STAT3 and ZEB1 proteins in E-cadherin down-regulation and human colorectal cancer epithelial-mesenchymal transition. J Biol Chem. 2012;287:5819-32.

\section{Submit your next manuscript to BioMed Central and take full advantage of:}

- Convenient online submission

- Thorough peer review

- No space constraints or color figure charges

- Immediate publication on acceptance

- Inclusion in PubMed, CAS, Scopus and Google Scholar

- Research which is freely available for redistribution

Submit your manuscript at www.biomedcentral.com/submit 